\title{
A successful case of cardiopulmonary arrest due to left ventricular free wall rupture treated with venoarterial extracorporeal membrane oxygenation and immediate induction of targeted temperature management
}

Ginga Suzuki ${ }^{1}$, Ryo Ichibayashi ${ }^{1}$, Saki Yamamoto ${ }^{1}$, Hibiki Serizawa ${ }^{1}$, Kota Kawada ${ }^{2}$, Toru Kameda $^{2}$, Yoshimi Nakamichi ${ }^{1}$, Masayuki Watanabe ${ }^{1}$, Shinnosuke Okuma ${ }^{2}$, and Mitsuru Honda $^{1}$

${ }^{1}$ Toho University Omori Medical Center

${ }^{2}$ Toho University

November 6, 2020

\begin{abstract}
We experienced a successful case of cardiopulmonary arrest due to left ventricular free wall rupture treated with venoarterial extracorporeal membrane oxygenation and immediate induction of targeted temperature management.
\end{abstract}

\section{Introduction}

Left ventricular free wall rupture (LVFWR) often occurs within 24 hours after myocardial infarction, and is a serious condition causing cardiac arrest due to cardiac tamponade [1]. As emergency measures, relieving the cardiac tamponade by pericardiocentesis and initiating venoarterial extracorporeal membrane oxygenation (VA ECMO) are considered. However, in cases of cardiac arrest, it is difficult to perform pericardiocentesis during chest compressions, and emergency left thoracotomy or initiating ECMO may be considered [2-4]. We experienced a case of cardiopulmonary arrest (CPA) due to LVFWR that was treated with VA ECMO and immediate induction of targeted temperature management (TTM).

\section{Case report}

A 73-year-old man lost consciousness and was transferred to our hospital. The patient had a history of testicular cancer treated with chemotherapy 2 years earlier and was in remission. He was barely able to communicate, and the complaint was unknown. Furthermore, he was in a shock with cold extremities. Peripheral intravenous infusion was initiated and transthoracic echocardiography revealed pericardial effusion and almost no systole. We decided to initiate ECMO based on a judgment of obstructive shock or cardiogenic shock. Cardiopulmonary resuscitation (CPR) was started because of CPA, before beginning ECMO. We also considered emergency left thoracotomy, but ECMO was prioritized. ECMO circulation was started 17 minutes after arrival and 10 minutes after CPA. Twelve-lead electrocardiography (ECG) revealed a QS pattern and ST elevation in leads V1-V5 (Fig. 1). We did not drain the pericardial fluid, but ECMO flow was stabilized using rapid infusion. The patient was immediately cooled with ECMO and reached a body temperature of $34^{\circ} \mathrm{C} 23$ minutes after CPA. He was then transferred for computed tomography (CT) for diagnosis. Contrast-enhanced CT showed no acute aortic dissection (AAD), and we diagnosed LVFWR due to myocardial infarction on electrocardiography. No leakage of contrast agent was apparent (Fig. 2). Blood tests revealed a troponin-I concentration of $13.4 \mathrm{ng} / \mathrm{ml}$ (Table); additional blood test results are shown in the 
Table. Emergency coronary angiography $(\mathrm{CAG})$ was performed to evaluate coronary artery lesions and to determine surgical procedures. In the CAG images, the \#7 branch of the left anterior descending coronary artery (LAD) was chronically totally occluded, and the \#4 branch of the right coronary artery provided collateral circulation to the LAD (Fig. 3). There was no other significant stenosis, and no rupture point could be found by left ventricular angiography. An intra-aortic balloon pump (IABP) was inserted under fluoroscopy, and the patient was immediately transferred to the operating room for surgical hemostasis.

Under general anesthesia, a skin incision was made from the sternal notch to the xiphoid process, and the pericardium was exposed by a median sternotomy. Leakage of bloody pericardial fluid was observed when the pericardium was incised. The pericardial fluid was aspirated to remove the blood clots in the pericardium, and oozing blood was observed near the cardiac apex (Fig. 4). The myocardium around the bleeding point had poor color tone, but no myocardial necrosis was observed in other areas. A total of five pieces of TachoSil@ (Takeda Pharmaceutical Company Limited, Tokyo, Japan) were attached, focusing on the bleeding site, and hemostasis was confirmed (Fig. 4). A towel was inserted into the pericardium to cover the bleeding area. Next, substernal and intrapericardial drains were inserted, and a 20-cc syringe was modified and used as a sternal bridge to prevent boney union. Four pieces of gauze were also inserted into the subcutaneous space. The skin was not sutured but instead, was covered with an Esmarch tourniquet and sutured, and a sterile drape was applied. Negative pressure was applied to the drain, and the chest was temporarily closed with a vacuum pack.

Considering the possibility of recurrent cardiac tamponade, the patient was admitted to the intensive care unit (ICU) with temporary chest closure. There was marked hemorrhagic drainage from the substernal and pericardial drains, and blood products were supplemented to stabilize the hemodynamics. TTM lasted 3 days, after which time we discontinued ECMO, but the consciousness disorder persisted. Waking on the sixth day of hospitalization, the patient was able to communicate. It was judged that the left ventricular assisting effect of the IABP had diminished, and we removed the device. On the same day, the amount of urine started to increase and diuresis began. Because edema of the mediastinal tissue and myocardium was controlled, we closed the chest incision on the 9th day of hospitalization. The patient was extubated on the 14th day of hospitalization, and on the 15th day of hospitalization, he was transferred to the general ward without neurological deficits. He is currently receiving rehabilitation.

\section{Ethical approval}

All subjects enrolled in this research have given their informed consent, which has been approved by my institutional committee on human and/or animal research, and this protocol has been found acceptable by them.

\section{Discussion}

In the present case, the keys to the patient's survival without neurological deficits were, first, the rapid initiation of ECMO for the CPA due to LVFWR, and second, we performed immediate TTM utilizing ECMO.

We would like to expand on the first point. Currently, there are three possible emergency treatments for CPA due to LVFWR, and the first is pericardiocentesis and drainage [1-4]. However, this procedure may be difficult to perform while continuing effective chest compressions. The second method involves pericardial drainage, primary hemostasis, and direct cardiac massage with left intercostal thoracotomy [4]. This method may be the quickest way to achieve drainage for a trained doctor. However, it may be difficult to identify the bleeding point, and if the point cannot be identified quickly, further bleeding may be promoted, and the patient may die. In addition, even if spontaneous circulation returns, this approach is highly invasive and may increase the risk of infection [5]. Moreover, when spontaneous circulation returns, ECMO may be necessary if cardiac function is severely deteriorated due to myocardial infarction. The third approach is to use ECMO $[2,3]$. We were able to establish ECMO as early as 10 minutes after CPA, as skilled doctors and clinical engineers were available continuously at our university hospital. As in the present case, when CPA occurs before a definitive diagnosis, AAD may be a cause of pericardial effusion [6], and lifesaving is expected to be 
difficult with left thoracotomy. Even if ST changes are detected on the ECG, these changes may occur with coronary artery dissociation, so definitive diagnosis before CT is difficult. ECMO flow may be insufficient for cardiac tamponade, but there is a possibility of survival with concomitant pericardiocentesis [6]. Even with CPA due to LVFWR, initiating ECMO is considered effective with skilled staff. Furthermore, introducing ECMO permits CAG. If there was stenosis in the remaining branch in our patient, coronary artery bypass surgery may have been added.

Regarding the second key point, in the present case, rapid ECMO enabled early introduction of TTM by rapid blood cooling. The patient's body temperature reached $34^{\circ} \mathrm{C} 30$ minutes after arrival and 23 minutes after CPA, suggesting the usefulness of ECMO from the viewpoint of brain protection. If resuscitation is performed with left thoracotomy, even if TTM is performed, an intravascular cooling device or body surface cooling is necessary [7], and it is difficult to introduce TTM promptly. Delayed brain protection can lead to post-cardiac arrest encephalopathy.

In conclusion, ECMO for CPA due to LVFWR is considered an effective resuscitation method because it enables rapid and minimally invasive cardiopulmonary support and rapid introduction of TTM.

\section{Disclosures}

\section{Acknowledgements}

We thank Jane Charbonneau, DVM, from Edanz Group (https://en-author-services.edanzgroup.com/ac) for editing a draft of this manuscript.

\section{Funding}

Nothing to declare.

\section{Conflict of interest}

The authors declare that they have no conflict of interest.

\section{Authors contributions}

G.S. wrote and drafted the manuscript. G.S., R.I., S.Y., H.S., Y.N., M.W., and M.H. helped to draft the manuscript. All authors read and approved the final manuscript

\section{References}

1. Purcaro A, Costantini C, Ciampani N, Mazzanti M, Silenzi C, Gili A, Belardinelli R, Astolfi D. Diagnostic criteria and management of subacute ventricular free wall rupture complicating acute myocardial infarction. Am J Cardiol 1997;80:397-405.

2. Arai R, Fukamachi D, Akutsu N, Tanaka M, Okumura Y. Surviving case of a blowout-type left ventricular free wall rupture during percutaneous coronary intervention for a lateral acute myocardial infarction. Int Heart J 2020;61:606-10.

3. Formica F, Corti F, Avalli L, Paolini G. ECMO support for the treatment of cardiogenic shock due to left ventricular free wall rupture. Interact Cardiovasc Thorac Surg 2005;4:30-2.

4. Ito M, Murayama H, Sudo Y, Ishida A, Asano S, Nakagawa Y, Nakamura T. Surgical repair of acute left ventricular free wall rupture: report of a case. Ann Thorac Cardiovasc Surg 2000;6:332-5.

5. Lau CK, Chin HF, Ong FHM, Eng KHA. Emergency department thoracotomy for pericardiac tamponade. Singapore Med J 2008;49:e382-4.

6. Yukawa T, Sugiyama K, Miyazaki K, Tanabe T, Ishikawa S, Hamabe Y. Treatment of a patient with acute aortic dissection using extracorporeal cardiopulmonary resuscitation after an out-of-hospital cardiac arrest: a case report. Acute Med Surg 2017;5:189-93.

7. Kalra R, Arora G, Patel N, Doshi R, Berra L, Arora P, Bajaj NS. Targeted temperature management after cardiac arrest: systematic review and meta-analyses. Anesth Analg 2018;126:867-75

\section{Figure Legends}


Fig. 1 The 12-lead electrocardiogram on arrival

A QS pattern and ST elevation were found in leads V1-V5.

Fig. 2 Contrast-enhanced CT on arrival

Pericardial fluid is seen in the images. The white arrow represents the extracorporeal membrane oxygenation cannula.

Fig. 3 Coronary artery angiography images on arrival

The \#7 branch of the left anterior descending coronary artery (LAD) was chronically totally occluded, and the \#4 branch of the right coronary artery provided collateral circulation to the LAD (white arrow).

Fig. 4 Intraoperative images

Oozing bleeding is visible near the cardiac apex (arrow). Chest closure was performed with a sutureless procedure.

\section{Hosted file}

Table.pdf available at https://authorea.com/users/373224/articles/491244-a-successfulcase-of-cardiopulmonary-arrest-due-to-left-ventricular-free-wall-rupture-treated-withvenoarterial-extracorporeal-membrane-oxygenation-and-immediate-induction-of-targetedtemperature-management

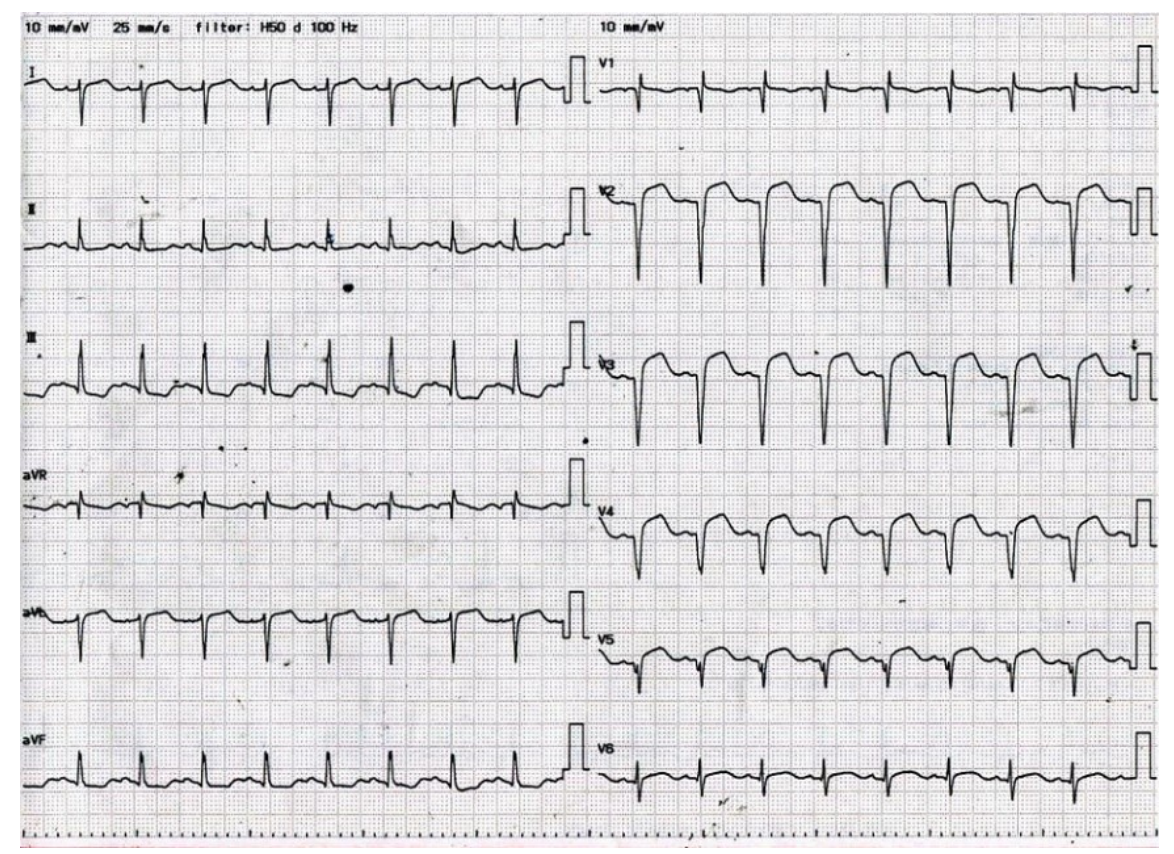



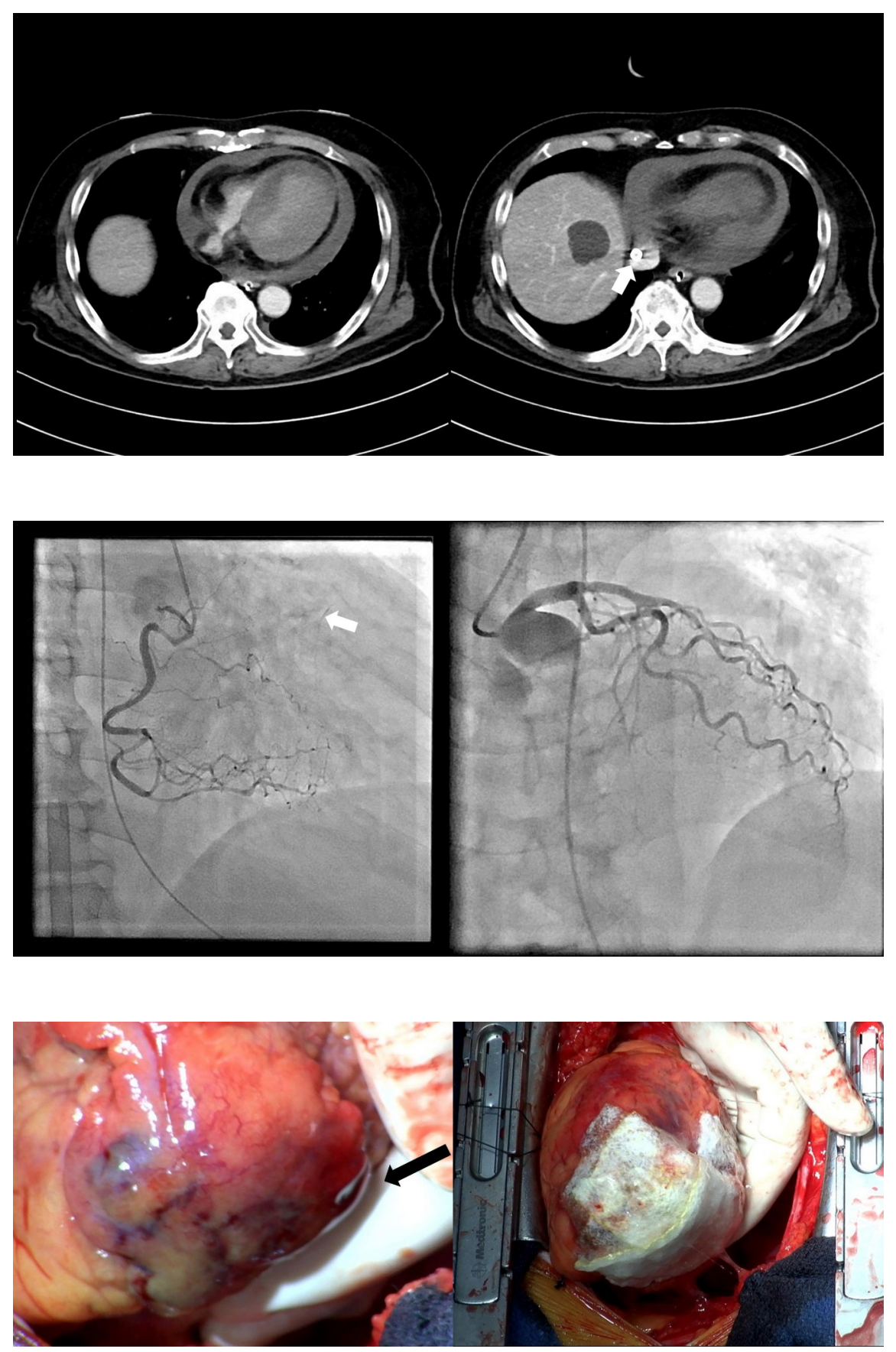